\title{
DIA-TORUS:A NOVEL TOPOLOGY FOR NETWORK ON CHIP DESIGN
}

\author{
Deewakar Thakyal and Pushpita Chatterjee \\ SRM Research Institute, Bangalore
}

\begin{abstract}
The shortcomings of conventional bus architectures are in terms of scalability and the ever increasing demand of more bandwidth. And also the feature size of sub-micron domain is decreasing making it difficult for bus architectures to fulfill the requirements of modern System on Chip (SoC) systems. Network on chip (NoC) architectures presents a solution to the earlier mentioned shortcomings by employing a packet based network for inter IP communications. A pivotal feature of NoC systems is the topology in which the system is arranged. Several parameters which are topology dependent like hop count, path diversity, degree and other various parameters affect the system performance. We propose a novel topology forNoC architecture which has been thoroughly compared with the existing topologies on the basis of different network parameters.
\end{abstract}

\section{KEYWORDS}

Network on chip, Torus, Topology, NoC

\section{INTRODUCTION}

Traditional bus architectures were working fine but then it started posing problems when too many deep sub-micron devices started showing disparities and the focus shifted to scalability. Scalability, being the primary goal, could not be achieved by bus architecture. These problems needed a well-structured approach, clear programming and modular design. This led to the rise of Network on Chip (NoC) architectures which offer promising solutions in terms of scalability and ease of communication. The paradigm has now shifted its focus from computation to communication and how it can cope with the scalability of the network. In most NoC architectures, the network is a set of connected router with processing nodes attached to those routers. Topology plays a crucial role in NoC, it defines how the cores are connected in a network[1]. Different IP cores can be arranged in different ways paving the way for a number of topologies that can be used for realizing a network. Topology affects the performance so it must be selected with care. So, a good topology is one which offers less hop count, more path diversity and can support load balancing. There are number of topologies in NoC which can be grouped as regular and irregular topologies. Regular topologies are those which follow a fixed pattern and can be divided into similar blocks. Irregular topologies don't follow any fixed arrangement of nodes.

In this paper we propose a novel topology, Dia-torus, for NoC systems. Present work aims at reducing the hop count and latency parameters. It has diametrical connections that enhance its performance. The proposed work is compared with the existing topologies. Different network parameters are used for the comparison. RTL implementation is also done for area and power

DOI: $10.5121 / \mathrm{ijcnc} .2016 .8310$ 
consumption analysis of the proposed work and other topologies. The related work with detailed description of the preliminary metrics for realizing a good NoC topology has been described in Section 2. Section 3 discusses the proposed Dia-Torus topology and routing procedure in the proposed topology. Weaddress the analysis and results that we get after comparing it with different topologies and depicted in a tabular form in Section 4 to show the efficacy of the proposed topology and conclude the paper by describing further future work in Section 5.

\section{RELATED WORK}

Topology designs become popular due to their simplicity and how easily they can be mapped using the fabrication techniques in practice. Some of these are mesh, torus and folded torus. Mesh is being used in number of NoC architectures namely, Intel's Teraflops containing 80 cores forming a 10x8 2D mesh[2], Tilera's 2D mesh [3] with 64 nodes in $8 \times 8$ mesh, the TRIPS processor [4] a wormhole routed 2D mesh with virtual channels. The complexity of router implementation depends on the degree of the router as more degree leads to more complexity.Number of buffers and ports required increases with increase in the number of degree. Topologies like torus[5], folded torus[6] and mesh [7], have uniform degree routers and are easy to implement whereas Dmesh[8], diametricmesh[9], Tmesh[10], xmesh, xtorus and xxtorus[11] all have no uniform degree of routers. The new torus network which has the hypercube Q3 as the basic module has been proposed[12]. The proposed Hyper-torus has the degree 4, and its network has the node and its edge symmetric and is scalable. The extra links and ports require more arbitration level and complex routing algorithms. Extra links in the topology can also help to increase the load balancing capabilities of the topology.

The performance analysis and comparison of $2 \mathrm{x} 4$ Network on Chip (NoC)topology has been done. First three common topologies, 2D Mesh topology, 2D Torus topology and hierarchical Mesh topology, are designed and then the performances of these three topologies are analyzed and compared in detail [12]. The simplicity of the regular mesh topology Network on Chip (NoC) architecture leads to reductions in design time and manufacturing cost but it's also unable to efficiently support cores of different sizes.

\subsection{Preliminaries}

A network topology comprises of different parameters and these parameters are gauged. On the basis of these parameters a topology is considered better than the other topologies. Various network parameters are explained in brief below.

- Degree: Degree of a router is the number of immediate neighbors it is connected to. In mesh topology, every router has a degree of four and only the edge routers have a degree of three. Degree of a router determines the ease of its implementation. Higher degree means the router has more number of nodes as neighbors which lead to increase in the number of ports resulting in more area and increase in implementation cost.

- Path Diversity: Path diversity is the number of shortest path between the two nodes in the topology. A network topology having more path diversity will be better equipped for load balancing. This results in better response to network congestion.

- Latency: Latency is the time taken by a packet to reach from its source to destination. Another parameter to measure latency is the average minimum latency which is the minimum of latencies of all the packets. Latency varies in a network due to congestion and traffic load. It gives the performance of the network under different traffic conditions. 
- Throughput: Throughput of the network is defined as the rate of data processed by a network in a given time and is represented as bits per second. Ideal condition in a network is of maximum throughput where all the channels are transferring data to the full capacity. It is also depended on other factors like flow control mechanisms and routing algorithms.

- Energy consumption: Transmission of packets through the network dissipates energy. Topologies with more hop links tend to consume more energy. Another source of energy consumption is the buffers in the router. Increasing temperatures can also give rise to hot spots and lead to wear and tear of the chip.

\subsection{NETWORK ON CHIP (NOC) TOPOLOGIES}

NoCsystems can be implemented with many different topologies. The choice of topology depends on the requirements and parameters like the complexity in implementing the topology, the area it requires and its routing algorithm.

1.Mesh: Mesh is the most frequently used and basic used topology with very simple routing algorithm. Mesh topology is easy to implement and is scalable. Except the corner and diagonal nodes, every node is connected to the four neighboring nodes. A diagonal node has degree 2 and the border nodes have degree 3 and all other nodes have degree 4 as shown in Fig.1. Mesh has a larger network diameter due to its more hop count. For a $4 \times 4$ mesh, the network diameter is 6 . The diagonal nodes are the one which contribute to more hop count (node 1 to 16). For routing, a XY routing algorithm can be used which routes packet based on the difference in co-ordinates.

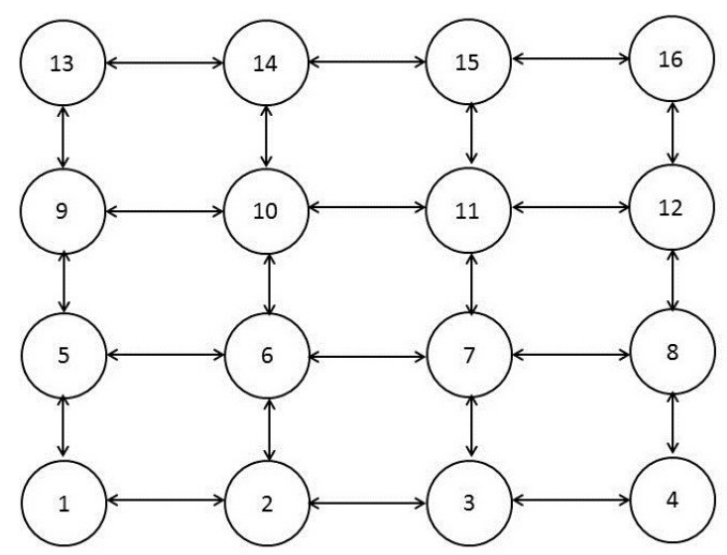

Figure 1. Mesh Topology

2.Torus:Torus is another topology that is popular because of its long wrap around links and is formed by connecting the boundary nodes in the same row and column as shown in the Fig.2. Torus has long end to end links which leads to less network diameter and less hop count. For a $4 \times 4$ torus topology, the network diameter is 4 as compared to 6 in mesh. In mesh it took 6 hops to reach diagonal nodes while it takes only 2 in torus. Torus requires long wires for the wrap around links when compared to mesh. 


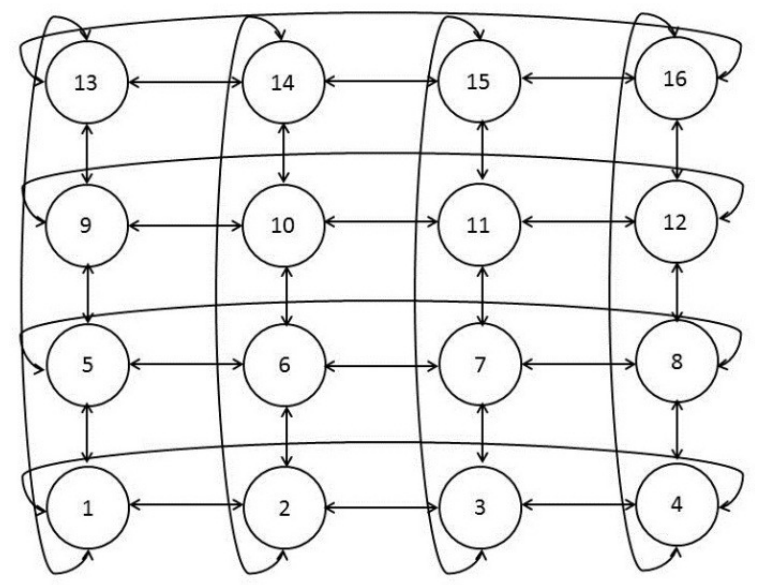

Figure 2. Torus Topology

3.Folded Torus:Another variant of torus is folded torus. Folded Torus has an advantage of shorter link length which helps to reduce the time packet required to traverse in the interconnected links. Shorter link length also contributes to the reduction in interconnect area required for implementation. Folded torus also has more path diversity than torus and is fault tolerant.

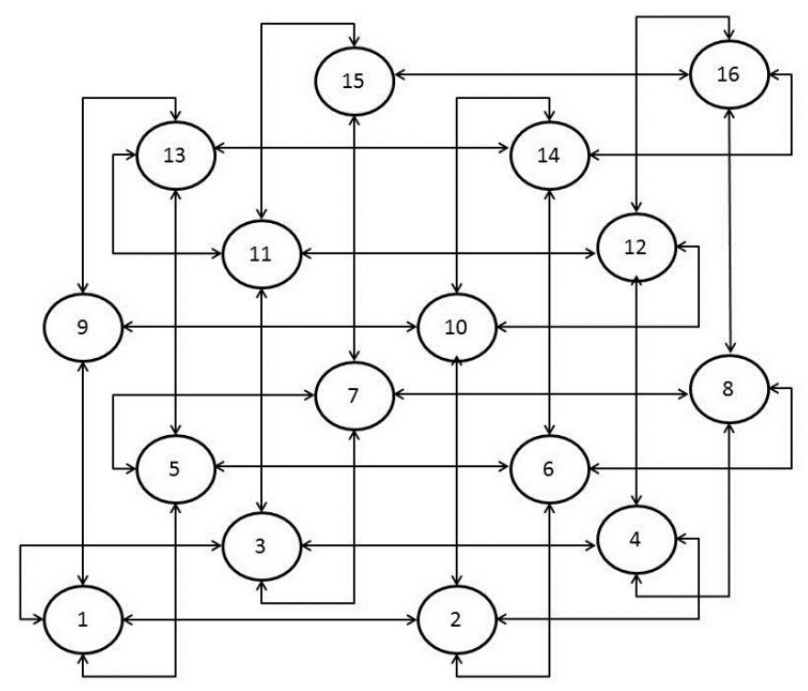

Figure 3. Folded Torus

4.Xmesh:Xmesh topology is an advancement of the existing mesh topology where it adds links in the diagonal direction to the mesh topology as shown in Fig.2 which results in the reduction of the diameter of the topology to half. In Xmesh topology, the hop count is also less than that of the mesh topology. The additional diagonal links contribute to increase in the area. Number of extra links added to xmesh topology is $n$ for anXn mesh topology. Xmesh has diameter 3 for a $4 x 4$ topology when compared to mesh which has a diameter value of 6 


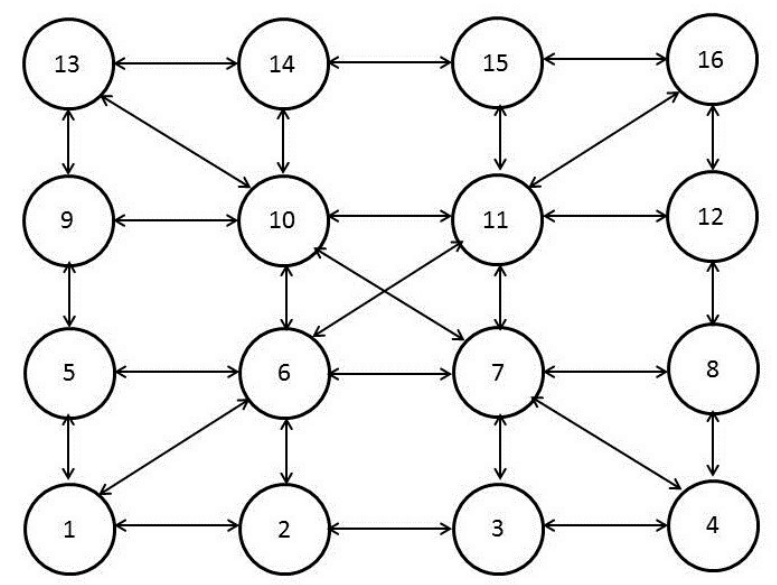

Figure 4.Xmesh Topology

5.Xtorus:Xtorus topology is also relatively new topology having diagonal connections where it increases the links in diagonal directions as done in Xmesh. Xtorus inherits the long warp around links of the torus topology. Diameter of Xtorus is better than torus whose diameter is $(n-1)$ when compared to that of torus $(n)$.

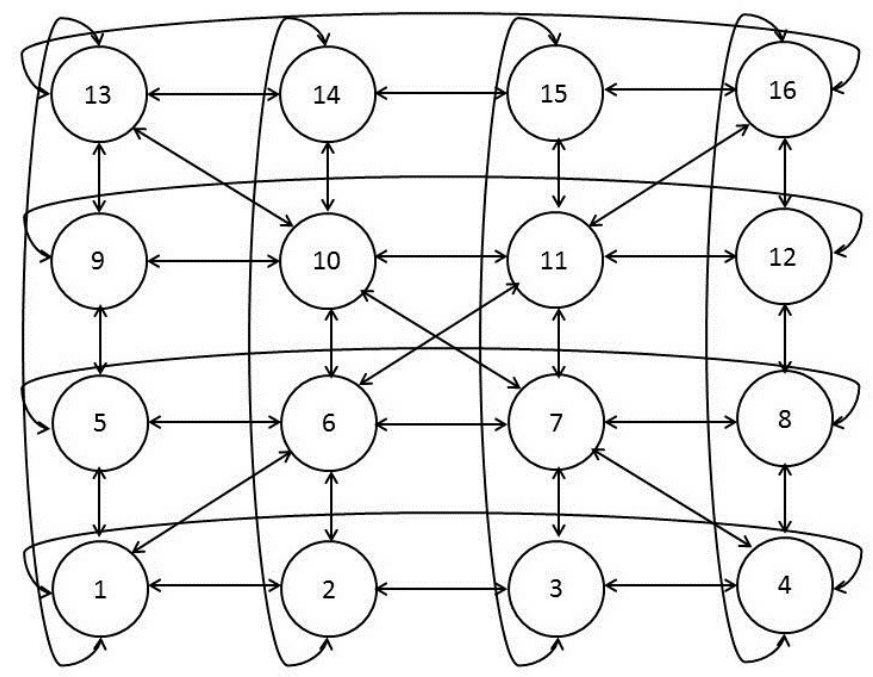

Figure 5.Xtorus Topology

6.King mesh:King mesh is a topology used is areas where parallel processing is a key aspect. In king mesh, every node is connected to its neighbor as shown in fig. Nodes that are in the center of the topology have higher degree than other nodes. Routers with higher degree have more area because of the extra buffers that are required for the extra ports that contribute to greater degree. Owing to greater number connections, the diameter of king mesh is less and the relative hoop count is also less. 


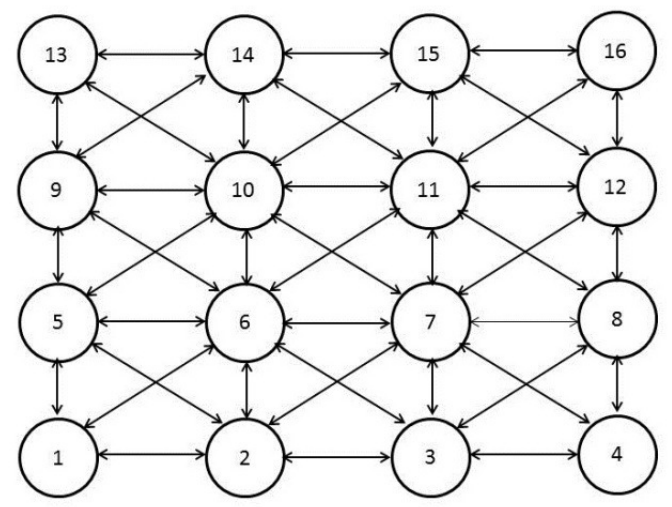

Figure 6. King Mesh Topology

7.King torus:King torus is another topology like king mesh. King torus, just as king mesh, is a torus topology having connections with all the neighboring routers. King torus will also have lager areas values than other regular topologies because of the extra ports in the routers. It also offers more in performance when compared to king mesh and other topologies as a result of the long connections between end to end nodes, both horizontally and diagonally.

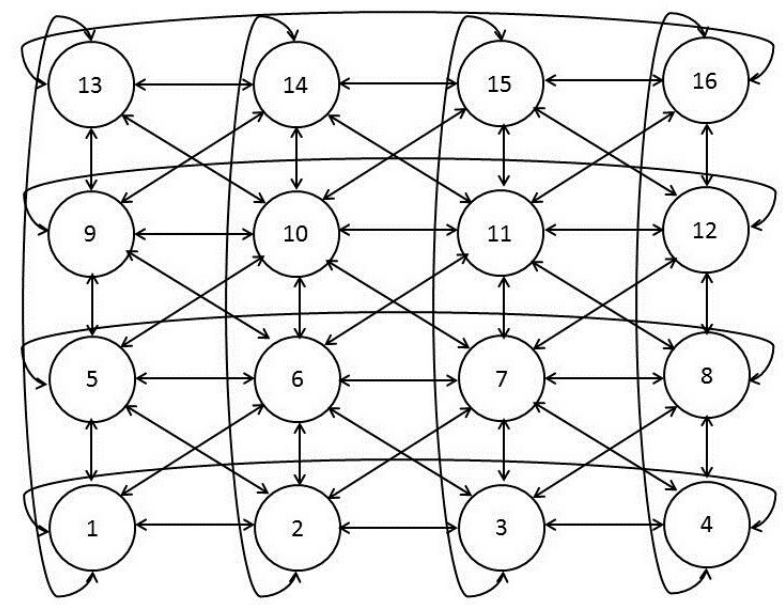

Figure 7. King Torus Topology

\section{Proposed WORK: DiA-TORUS TOPOLOGY}

In this paper, we propose a new topology, Dia-Torus, which is a hybrid topology with features of both mesh and torus topologies. In addition to the long vertical and horizontal links that torus has, Dia-Torus has diagonal links that connect the four edge nodes with the nodes in the second last row of the topology as shown in Fig 4. The edge routers having diagonal links do not need an additional port. Only the four nodes need 5 port routers that are connected with those diagonal links. Since only four 5 port routers are used irrespective of the size of topology, there is only slight increase in the area. The diagonal links help in reducing the hop count by a significant value. Moreover, the diameter of the topology is also reduced because of the presence of these links. In the Fig.4, nodes 1, 4, 13, 16 are edge nodes and nodes 1 to 4, 5, 8,9,12 and 13 to 16are border nodes. 


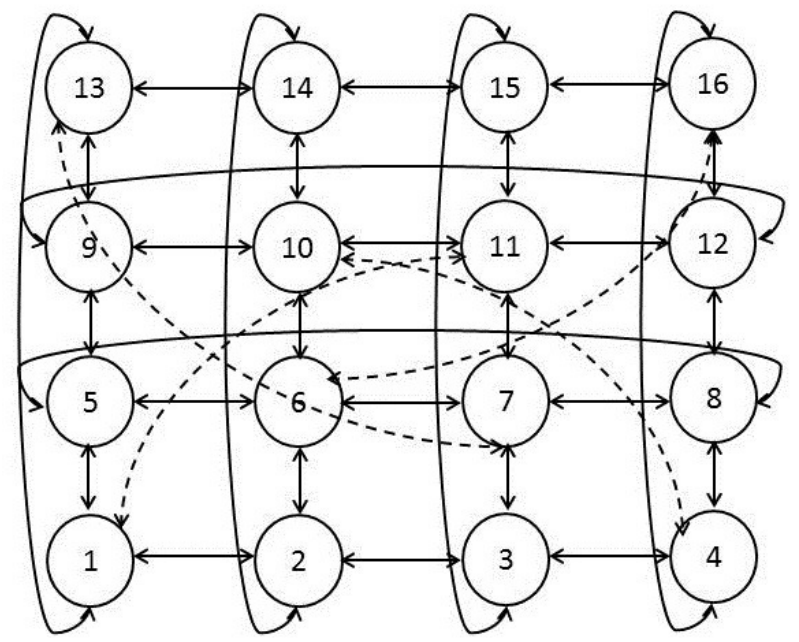

Figure 8.Dia-Torus Topology

\subsection{ROUTER ARCHITECTURE}

Network on chip (NoC) architectures are implemented as tile based structure to ease on chip communications [13][14][15]. A tile can be a general purpose processor, a memory subsystem etc. A router is attached which connects this tile to neighboring nodes. All communications with the other nodes in the architecture are done via this router. Figure 9(a) shows the tile structure and basic router architecture is shown in Figure 9(b). The router has 4 named on directions as east, west, north and south. There is another port, local port, dedicated port for communication with the processing core. Buffers are usually attached to store incoming data
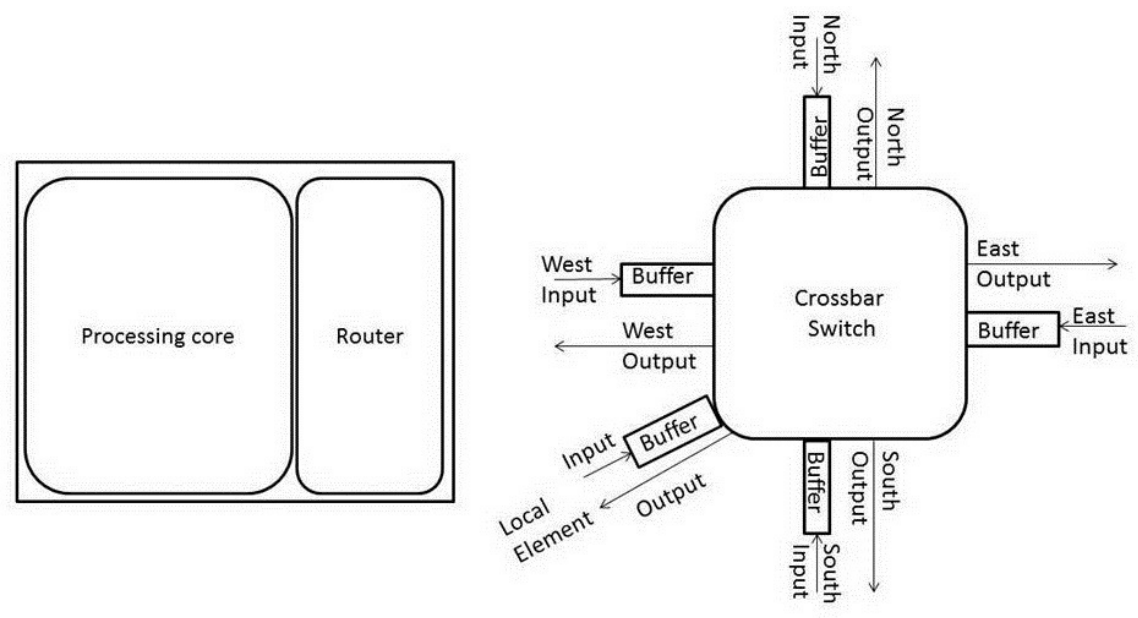

Figure 9:(a) NoC tile with processing core and router. (b) Noc 4 port router

Present work has a router with an additional port for the diagonal connections. There are only 4routers in the topology that have an extra port. The extra port leads to a slight increase in area of the router but it also manages to route packets efficiently reducing the overall hop count. The router structure is shown in figure 10. 


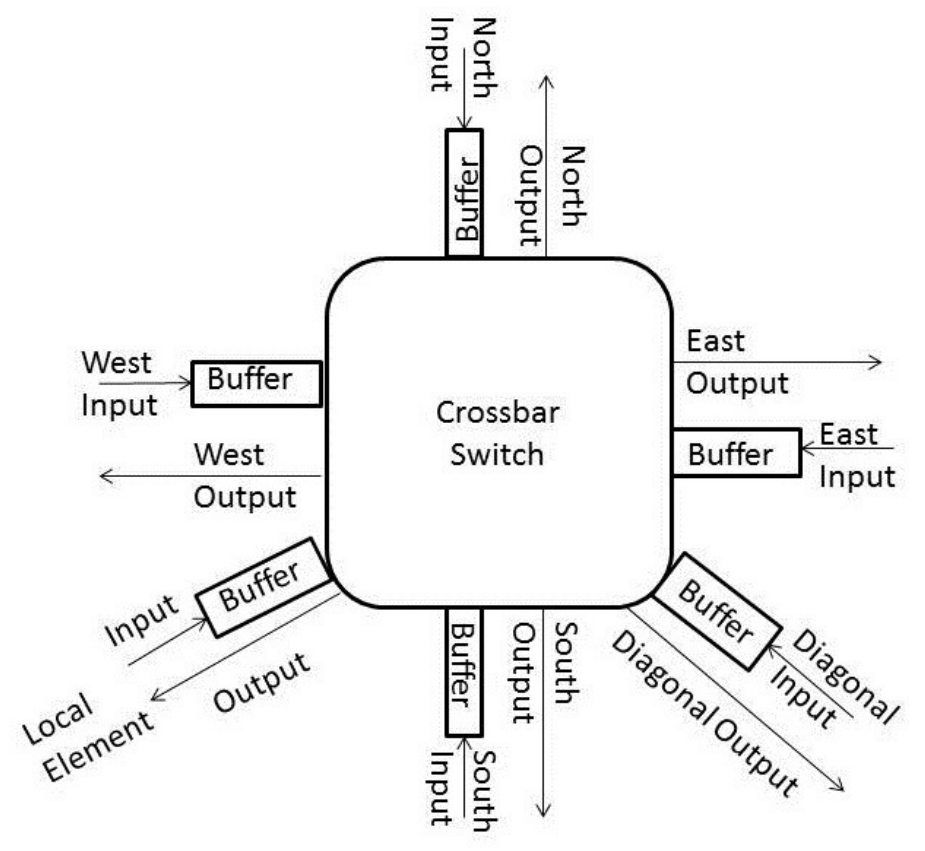

Figure 10. 5 port router used in Dia-torus

\subsection{Routing Algorithm}

The routing algorithm for the present work is based upon the routing algorithm for torus. Additional conditions necessary for the topology are added. It has four routers that have an extra port named diagonal port for long diametrical connections. Edge routers have diametrical connections attached to the existing port only and no extra port is added. For anxnDia-torus the routing algorithm is as follows

1. If the source node is a border node:

a. If source is an edge node and destination is in the opposite quadrant with $\mathrm{x}$ and $\mathrm{y}$ offset greater than $\mathrm{n} / 2$, take the diametrical link;

b. For destination nodes with hop count less than that, routing is done as in the case of torus by taking the wrap around links in considerations to hop farther nodes and normal XY for rest of the nodes.

i. Exceptions are the four edge nodes don't have horizontal wrap around links so additional condition for packet to move are written.

c. For all border nodes except the edge nodes, routing algorithm is same as that of torus for both offset less than $\mathrm{n} / 2$. Else nearest router with diametrical link is taken.

2. For nodes that are not border nodes, if both $\mathrm{x}$ and $\mathrm{y}$ offset are greater than $\mathrm{n} / 2$,

a. Go to the nearest node with a diametrical link, 5 port router, and move directly.

b. Else routing algorithm is same as torus. 
International Journal of Computer Networks \& Communications (IJCNC) Vol.8, No.3, May 2016

\section{RESULTS AND COMPARISON}

The proposed work is compared with different topologies on the basis of several network parameters. The topologies mentioned in the preliminaries are used for the comparison.

- Hop Count:Hop count is the number of nodes that a flit traverses from its source to destination. Dia-Torus has a significant decrease in hop count when compared with the existing topology. The reduction in hop count when compared with the existing topologies is: $31 \%$ for mesh, $13 \%$ for torus, $5.1 \%$ for twisted torus. Table 1 shows the hop count comparison of different topologies with Dia-Torus.

- Network Diameter:Network Diameter is to the largest, minimal hop count over all pairs of nodes in the network. In anxn network, the diameter ofmesh is $2(n-1)$, for xmesh its $n-1$. When $n$ is odd, the diameter of torus is $n-1$ and $n$ for even values. The network diameter ofxtorusisn-1. For king mesh and king torus diameter is $n-1$. Dia-torus also has diameter of $n-1$. Table 1 compares the diameter value of the topologies.

- Ideal Average Latency: For a given topology, assuming that there is no congestion in the network, the average delay between all the nodes in the topology is called the ideal average latency $(T)$. It can be calculated by the formula

$$
T=H T_{r}+\frac{D}{v}+\frac{L}{b}
$$

Where $H$ is the average hop count from source node to destination node, $T_{r}$ is the delay in routing on the router, and the unit is cycle/hop,Dis the average distance from the source node to destination node, which usually equals to $H$, and the unit is hop. vis the wire transmission speed, and the unit is hop/cycle, $L$ is the packet length, and the unit is flit. bis the bandwidth, and the unit is flit/cycle. Generally, different topologies correspond to different $H$ and $D$ values, while $T_{r}$ is dependent on the routing algorithm and the physical implementation of the router.From the Table 1 , it can be seen that latency value for proposed work is $26.4 \%$ less than that of mesh, $11.08 \%$ less than torus, $3.8 \%$ less than that of twisted torus. It also has less latency value compared to that of Xmesh, $6.1 \%$ less and equal to xtorus but with less number of links.

- Ideal Throughput: For a given topology, ideal throughput is the maximum network throughput in a perfect flow control and routing mechanism. William James Dally, et al., gave a formula [1]for calculating the ideal throughput $(T H)$. Bcis the number of channels required to divide the whole network into two equal halves; $b$ is the width of each channel, $N$ is the number of nodes.

$$
T H<=2 b^{*} B c / N
$$

Taking $4 \times 4$ node scale for example, for mesh, it takes four links to divide it into two halves and each link is bidirectional, so $B c=8$. In the same way, it is easy to get that $B c$ for torus,xmesh,xtorusand Dia-Toruswhich equals to 16, 12, 20 and 20 respectively.Assuming that all other parameters are constant, the ideal throughput of Dia-Torus increases by $150 \%$ when it is compared with that of mesh,and increases by $25 \%$ compared with that of torus and $60 \%$ xmesh.

- Path diversity:Path diversity is the property of a topology to possess more than one shortest path between two nodes. With better path diversity, it is possible to select between more multiple shortest paths, which can effectively reduce network congestion and enhance network fault tolerance. 
International Journal of Computer Networks \& Communications (IJCNC) Vol.8, No.3, May 2016

Table I: Table showing comparison of proposed work with different topologies

\begin{tabular}{|c|l|l|l|l|l|l|l|l|}
\hline Topology & Mesh & Torus & $\begin{array}{l}\text { Folded } \\
\text { Torus }\end{array}$ & $\begin{array}{l}\text { King } \\
\text { Mesh }\end{array}$ & $\begin{array}{l}\text { King } \\
\text { Torus }\end{array}$ & $\begin{array}{l}\text { Xmes } \\
\mathrm{h}\end{array}$ & Xtorus & Dia-Torus \\
\hline Hop Count & 644 & 514 & 468 & 456 & 396 & 460 & 444 & 444 \\
\hline Diameter & 6 & 4 & 4 & 3 & 3 & 3 & 3 & 3 \\
\hline Latency & 14.5 & 12 & 13.32 & 10.9 & 10.75 & 11.37 & 10.67 & 10.67 \\
\hline $\begin{array}{c}\text { Path } \\
\text { Diversity }\end{array}$ & 2.96 & 2 & 2.24 & 4.14 & 4.34 & 1.4 & 2.08 & 1.62 \\
\hline
\end{tabular}

- Physical Implementation Difficulty and Area:The physical implementation difficulty of different topologies depends on the symmetry of the structure, the number of dimensions, the number of long edges, the number of ports of each router and the area occupied by the topology. More the number of ports in the router more are the buffer requirements which increase the area of the router. Table II and Table III describes the comparison between different topologies with respect to number of links and area consumption.

Table II: Table showing number of links comparison of proposed work with different topologies

\begin{tabular}{|l|l|l|l|l|l|}
\hline Topology & Mesh & Torus & Xmesh & Xtorus & Dia-Torus \\
\hline Number of links & $2 \mathrm{n}(\mathrm{n}-1)$ & $2 n^{2}$ & $2 n^{2}$ & $2 n^{2}+2(\mathrm{n}-1)$ & $2 n^{2}+2$ \\
\hline
\end{tabular}

Table III: Table showing area comparison of proposed work with different topologies

\begin{tabular}{|l|l|l|l|l|}
\hline Topology & Mesh & Torus & Folded Torus & Dia-Torus \\
\hline $\begin{array}{l}\text { Total } \\
\text { area }\left(\mu \mathrm{m}^{2}\right)\end{array}$ & 2703521.651525 & 2875182.661041 & 2883018.998952 & 2891311.592451 \\
\hline
\end{tabular}

- Energy consumption: Power consumed by packets is another factor that affects network performance. Table IV shows the power consumed by different topologies under same traffic conditions. As can be seen, the present work consumes less power than mesh and torus topologies.

Table IV: Table showing energy comparison of proposed work with different topologies

\begin{tabular}{|l|l|l|l|l|}
\hline Topology & Mesh & Torus & Folded Torus & Dia-Torus \\
\hline $\begin{array}{l}\text { Total Power } \\
\text { consumed }(\mu W)\end{array}$ & $1.4889 \mathrm{e}+04$ & $1.5121 \mathrm{e}+04$ & $1.1415 \mathrm{e}+04$ & $1.4696 \mathrm{e}+04$ \\
\hline
\end{tabular}




\section{CONClusions And Future Works}

From the above results it can be concluded that the proposed work offers significant advantages in hop count and diameter.Dia-torus also offer more ideal throughput when compared to existing topologies. Latency of the present work is also less. It is comparable with latency values of king torus and xtorus which have more number of links than the proposed work.Proposed work has a trade off in terms of path diversity. When compared with mesh, torus and othertopologies, proposed work loses some path diversity. The proposed topology has a slight increase in the area which is negligible when the advantages are considered. Routing algorithm for the proposed work doesn't handle faulty links. A new fault free and deadlock free routing algorithm will be developed in near future. Also, the proposed topology will be implemented in ASIC.We are working on implementing the proposed topology on NoC simulator Noxim [14] and the functionality of Noxim [15] will be extended for evaluation of faulty links in a network and network simulation will be done with those faulty links and how the performance is affected.

\section{REFERENCES}

[1] W. J. Dally, B. Towles, Principles and Practices of Interconnection Networks, Morgan Kaufmann Pub., San Francisco, CA, 2004.

[2] S. Vangal et al, "An 80-Tile 1.28TFLOPS Network-on-Chip in 65nm CMOS”, In proceedings of IEEEInternational Solid-State Circuits Conf., Digest of Tech. Papers (ISSCC),pp. 98-10, 2007.

[3] A. Agarwal, L. Bao, J. Brown, B. Edwards, M. Mattina, C.C. Miao, C. Ramey, D. Wentzlaff, "Tile Processor: Embedded Multicore for Networking and Multimedia", In proceedings of HotChips19, Stanford, CA, Aug. 2007.

[4] P. Gratz, C. Kim, R. McDonald, S. W. Keckler, D. C. Burger, "Implementation and evaluation of onchip network architectures", In proceedings of Int. Conf. Computer Design,pp.477-484,2006.

[5] Kumar,S, Jantsch,A, Soininen,J.P, Forsell,M, Millberg,M, Oberg,J, Tiensyrja,K, Hemani,A, “A network on chip architecture and design methodology". In proceedings of IEEE Computer Society Annual Symposium onVLSI,pp.105-112,2002.

[6] W. J. Dally and C. L. Seitz., "The Torus routing chip", Journal of Distributed Computing, pp.187$196,1986$.

[7] W. J. Dally and B. Towles., "Route packets, not wires: on-chip interconnection networks", In proceedings of DAC,pp. 684-689,2001.

[8] Chifeng Wang, Wen-Hsiang Hu, SeungEun Lee, Nader Bagherzadeh, "Area and power-efficient innovative congestion-aware Network-on- Chip architecture", Journal of Systems Architecture, pp.24-38,2011.

[9] M. Reshadi, A. Khademzadeh, A. Reza, M. Bahmani, "A Novel Mesh Architecture for On-Chip Networks", Design and Reuse Industry Articles,2013.

[10] Quansheng Yang, Zhekai Wu, “An Improved Mesh Topology and Its Routing Algorithm for NoC”,In proceedings of International Conference on Computational Intelligence and Software Engineering (CiSE), (2010), pp:1-4.

[11] Liu Yu-hang, Zhu Ming-fa, Wang Jue, Xiao Li-min, Gong Tao, "Xtorus: An Extended Torus Topology for On-Chip Massive Data Communication", In proceedings ofIEEE 26th International Symposium Workshops \& PhD Forum on Parallel and Distributed Processing,pp.2061-2068,2012.

[12] Woo-seo Ki, Hyeong-Ok Lee, Jae-Cheol Oh, "The New Torus Network Design Based On 3Dimensional Hypercube",In proceedings of 11th International Conference on Advanced Communication Technology, ICACT 2009, Vol:01, 2009.

[13] A. Hemani et al., "Network on a chip: An architecture for billion transistor era," in Proc. IEEE NorChip Conf., Nov. 2000, pp. 166-173.

[14] S. Kumar et al., "A network on chip architecture and design methodology," in Proc. Symp. VLSI, Apr. 2002, pp. 105-112.

[15] W. J. Dally and B. Towles, "Route packets, not wires: on-chip interconnection networks," in Proc. Design Automation Conf., Jun. 2001, pp.684-689 
[16]J uyeob Kim, Miyoung Lee, Wonjong Kim, Junyoung Chang, YounghwanBae and Hanjin Cho "Performance analysis of NoC structure based star mesh topology",In proceedings of SoC Design Conference, ISOCC '08, Vol:02, 2008.

[17] F Fazzino., M Palesi., D Patti., "Noxim: Network-on-Chip Simulator", url: http://sourceforge.net/projects/noxim/ accessed on April, 2016.

[18] K. Swaminathan, D. Thakyal, S. G. Nambiar, G. Lakshminarayanan and Seok-Bum Ko, "Enhanced Noxim simulator for performance evaluation of network on chip topologies," In proceedings of Recent Advances of Engineering and Computational Sciences (RAECS), 2014, pp. 1-5, 2014.

\section{AuThors}

Pushpita Chatterjee is working as a research lead at SRM Research Institute, Bangalore since 2012. She completed her PhD from Indian Institute of Technology Kharagpur, India in 2012. She has over 15 publications to her credit in international journals and conferences. Her research interests are mobile computing, distributed and trust computing, wireless ad hoc and sensor networks, network on chip, information-centric networking an software-defined networking.

Deewakar Thakyal is working as a research member at SRM Research Institute, Bangalore since 2015. He received his Master's degree from National Institute of Technology, Tiruchirappalli, India in 2014. His research interests are network on chip, internet of things, sensor networks and cyber physical systems.
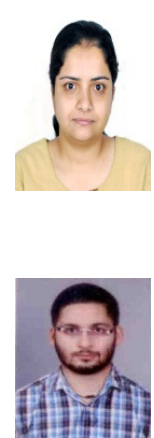